smoothed and anisotropically etched samples was evident throughout a wide range of waveguide widths. According to the researchers, this smoothing technology could lead to significant improvements in practical waveguide design for optical devices.

Tim PALUCKA

\section{Dynamics Study of Polymer Chains Shows Reduction in Atomic Diffusion Coefficients at a Free Surface}

In the November 12, 2001, issue of Physical Review Letters, researchers from the State University of New York-Stony Brook reported the relationship between structure and dynamics of polystyrene (hPS) by dynamic secondary-ion mass spectrometry (DSIMS). Their experimental results confirmed the chain-conformation calculation at the free surface by meanfield theory.

In order to understand how the center of mass diffusion of polystyrene related to its different molecular weight as a function of distance from the vacuum interface, a special multiple-layer sample used in the DSIMS experiment was designed. It had two parts, each consisting of a 200-nm layer of matrix hPS followed by a $25-\mathrm{nm}$ layer of $20 \%$ monodisperse deuterated analogue (dPS) and 80\% matrix hPS. From the relationship between the concentration of the tracer polymer (hPS or dPS) volume and the distance from the free surface for the four-layer sample, the diffusion coefficients for diffusion into a semi-infinite and an infinite medium for the top layer and the middle layer were both reduced relative to the bulk value within the distance from the surface.

The researchers found that "a purely enthalpic argument" cannot explain the experimental result. They had previously reported a large slowing down of dynamics when polymer chains were oriented parallel to the surface by rubbing the surface. They then hypothesized that the stringent confinement imposed by the large surface tension at the vacuum interface was inducing the chain orientation. This type of orientation was also plausible from the theoretical work of Kurt Binder, who demonstrated that the fluctuation times at the surface of a polymer film were anisotropic - namely, those parallel to the sample surface were much faster than those in the perpendicular direction. To test this hypothesis, self-consistent field theory was used and a one-dimensional equilibrium lattice method was designed, in consideration of the small system size. This method identified the segmental distribution at the surface, that is, the number of segments a chain has near the surface as a function of its position from the surface. After comparing the theoretical calculation with the experiment results of the chain dynamics, the researchers confirmed that asymmetric segmental fluctuations at the surface resulted in decreased mobility at the surface. This result overturned the long-held assumption that a large enhancement in mobility would be found at the surface, in analogy to monomolecular systems where this effect was observed as a result of the reduced potential.

Yue Hu

\section{One-Dimensional Metallic Edge States Observed in $\mathbf{M o S}_{\mathbf{2}}$}

Researchers at the Technical University of Denmark and the University of Aarhus have found metallic states along the edges of two-dimensional $\mathrm{MoS}_{2}$ clusters. These edge states are a realization of one-dimensional conducting wires and have been observed in $\mathrm{MoS}_{2}$ nanoclusters on $\mathrm{Au}(111)$ substrates.

M.V. Bollinger, J.V. Lauritsen, and coworkers described their results in the November 5, 2001, issue of Physical Review Letters. The researchers performed density functional theory (DFT) calculations on a single layer of $\mathrm{MoS}_{2}$ and studied the edges in a model system consisting of a stripe of $\mathrm{MoS}_{2}$ in a supercell geometry. They also reported experimental scanning tunneling microscopy (STM) images of $\mathrm{MoS}_{2}$ nanoclusters on $\mathrm{Au}(111)$ substrates along with simulated STM images calculated from a Tersoff-Hamann model.

The experimental STM images achieve atomic resolution and reveal the detailed structure of these $\mathrm{MoS}_{2}$ clusters. The images also show a brim of high conductance that extends around the edge of these clusters.

The DFT calculations for $\mathrm{MoS}_{2}$ edges show two one-dimensional metallic states localized at the molybdenum edge. One of these states (edge state I) is localized at adsorbed sulfur dimers on this edge and is a superposition of $p_{x}$ orbitals extending in two parallel chains.

To investigate whether these metallic edge states are responsible for the conducting brims seen in experiments, the researchers simulated STM images for $\mathrm{MoS}_{2}$ stripes with and without an underlying $\mathrm{Au}(111)$ substrate. These calculations showed that the brim is due to the $p$ orbital arising from the $p-d$ bond between sulfur and molybdenum.

"The one-dimensional metallic wires existing at the edges of the $\mathrm{MoS}_{2}$ nanoclusters may provide a template for investigating the properties of coherent electrons on this length scale," said Bollinger.

Another important consequence of this work, according to the report, is a better understanding of the catalytic properties of $\mathrm{MoS}_{2}$. Bollinger said, "It is generally accepted that catalytically active sites of $\mathrm{MoS}_{2}$ are localized at edges. Since the electronic structure at edges determines this reactivity, it is certainly possible the metallic edge states play an important role in this context."

\section{CHRISTOPHER MATRANGA}

\section{Low-Temperature Aging of Zirconia Ferrules Limits Optical-Connector Reliability}

The increased use of optical fibers in uncontrolled environments has recently led to research focused on the durability and reliability of interconnects. Current technology employs flat-end-face connectors where the reduction of reflected light at the fiber end is achieved by physical contact between the optical fibers secured in a stabilized zirconia ferrule. A team of researchers from the Oak Ridge National Laboratory, Rutgers University, and Bellcore in Morristown, N.J. has shown that strict polishing, which ensures physical contact, accelerates the aging of zirconia ferrules by relieving compressive stress, making the resulting phase transformation more thermodynamically favorable. This environmental-aging-induced phase transformation results in a 4-5\% increase in the unit-cell volume, leading to surface roughening and optical losses due to contact problems, thus limiting connector lifetimes.

As reported in the November 2001 issue of the Journal of the American Ceramics Society, the research team, led by Michael Lance, aged samples from a variety of manufacturers at temperatures from $-10^{\circ} \mathrm{C}$ to $85^{\circ} \mathrm{C}$ at $95 \%$ relative humidity for 90 days. Studying the results with profilometry, optical, and interferometric microscopy, the researchers found two patterns. In some samples, they observed a significant increase in the surface roughness of polished zirconia ferrules, corresponding to a $1-2 \mathrm{vol} \%$ phase transformation. In other samples, they saw a flattening or deformation of the zirconia surface, corresponding to an increased volume content of monoclinic transformed zirconia, up to $40 \mathrm{vol} \%$.

Raman spectroscopy equipped with a confocal aperture was used to control the sampling depth and to quantify the tetragonal-monoclinic phase transformation. They reported that the amount of transformation was lower in the vicinity of the optical-fiber-region under compression-and that transformation occurred only at the polished surface of the material. In addition, no monoclinic zirconia was found at the sides of the fer- 
rules that were not polished and still under compression from the machining process. A large variation in performance was observed among zirconia ferrules from different manufacturers with different processing procedures. They attribute this variation to surface deformation and suggest that the subsequent decrease in light transmission may be minimized with properly identified polishing procedures that would limit the amount of aging-induced phase transformation at the polished ferrule surface.

KYLE BRINKMAN

\section{In Situ Stone-Age Bone-Tool Industry Uncovered}

Researchers from Iziko South African Museum in Cape Town; the Institut de Prehistoire et de Geologie du Quaternaire in Talence, France; the Institute of Human Origins at Arizona State University (ASU); and Chicago State University have dated a large set of specialized bone tools at more than 70,000 years old. Found with related artifacts in the Blombos Cave, which is set in a cliff overlooking the Indian Ocean at the extreme southern tip of South Africa, the tools represent evidence that modern human behaviors evolved much earlier than previously thought.

The researchers report in the December 2001 issue of the Journal of Human Evolution various findings that date the group of 28 bone tools. They said that bifacially flaked stone points, possibly used as spearheads, occur above and within the layers in which the bone tools were found. Archaeological sequences from other sites with bifacial points in the southern Cape date to about 65,000 years ago, suggesting that the bone tools at Blombos are at least that old.

A distinct, sterile, yellow sand horizon lies above dark, anthropogenic (humanproduced) sediments that contain the bifacial points and the bone tools, and below dark, anthropogenic sediments of more recent age. The sand layer acts as a clear stratigraphic break because any penetration through this yellow sand by younger materials from above would be clearly visible; the sand layers are clearly undisturbed above where many of the bone tools occur, the researchers report.

At other archaeological sites in South Africa, such as Die Kelders Cave 1, similar dune layers are found that date to the last glacial period (roughly 60,000-70,000 years ago), when the ocean withdrew from the current coastline. On geological grounds, the researchers believe that the sand layer at Blombos is of similar age, making the bone tools somewhat older. Using thermoluminescence methods, the research team is in the process of directdating these sterile yellow dune sediments and burnt stone from the same layers as the bone tools.

The existence of bone and other "formal" tools has been frequently cited as an important item on the trait list of archaeological details traditionally seen as indicators of the presence of modern human behavior in Stone Age and Upper Paleolithic populations. Other items on the list include the hunting of large fish, the use of decoration, and the production of artevidence of symbolic thinking.
The excavations at Blombos Cave and similar sites have also yielded substantial quantities of ochre, a mineral compound that aboriginal peoples frequently use for body decoration.

Christopher S. Henshilwood, affiliate archaeologist at Iziko and adjunct associate professor of archaeology at the State University of New York-Stony Brook, said, "Based on extensive evidence from European sites, what has been cited repeatedly is that there was an Upper Paleolithic 'symbolic explosion' that archaeologists have said indicates the best recorded beginning of modern human behavior at about 35,000 years ago. We're seeing evidence of a comparable change in Africa, but in the Middle Stone Age-more than twice as far back in time." Earlier, Henshilwood reported on a fragment of "deliberately engraved bone" found at the site.

Curtis W. Marean of ASU said, "The African record is starting to show evidence that is making it look fundamentally different from Eurasia at the time. We're getting a lot of evidence for symbolizing behavior, and that is almost certainly related to language."

\section{FOR MORE RESEARC'J NEMVS ON MASJERJALS SCJENCE . . \\ ... access the Materials Research Society Web site: \\ www.mrs.org/gateway/ matl_news.html}

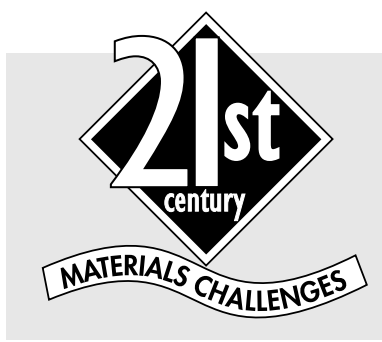

The materials field has developed from one of finding and using materials to one where the materials essentially can be designed and built atom by atom. The hunter has become the tiller. What will the next century uncover as we dig deeper?

View Articles on Materials Challenges for the Next Century www.mrs.org/publications/bulletin/21stcen

\begin{tabular}{|c|c|c|c|}
\hline \multicolumn{4}{|c|}{ Advertisers in This Issue } \\
\hline & Page No. & & Page No. \\
\hline Bede Scientific & 54 & Huntington Mechanical Labs, Inc. & Outside back cover \\
\hline Brookhaven National Lab/NanoCenter & 4 & Hysitron, Inc. & 18 \\
\hline Chemat Technology, Inc. & 29 & IUMRS Facets & 55 \\
\hline CSIRO & 51 & MTS Systems Corporation & 38 \\
\hline High Voltage Engineering & Inside front cover & Society of Vacuum Coaters & 75 \\
\hline
\end{tabular}

